\section{MECHANICAL PROPERTIES OF METALS}

\section{CONFERENCE AT BRISTOL}

\begin{abstract}
A SUMMER school and conference on the mechanical properties of metals was held at the University of Bristol during July 2-9. At the summor school (July 2-5) a course of lectures was given by Prof. N. F. Mott, Mr. F. R. N. Nabarro and some of their colleagues on the theory of the subject, especially on dislocations, plastic flow and precipita. tion. During the conference, papers mainly on experimental topics were given by physicists from various countries, including Meijering (oxidation hardening), Weil (ordered state in alloys), Druyvesteyn (mechanical properties at low temperatures), Andrade, Orowan, Chalmers, Cottrell (creep), Shockley (slip planes in silver halides), Lacombe (crystal boundaries), W. G. Burgers (recovery and recrystallization), Crussard (diffusion studied by thermoelectric methods), Guinier (precipitation), Snoek (elastic after-effects in iron), Guillet (internal friction), $\mathrm{McKe}$ and Leadbeater (powders).

Some of this work has already appeared in scientific journals, and many of the papers will be published in a special number of the Proceedings of the Physical Society; in this article it seems, therefore, more useful to pick out some salient points from the conference rather than to attempt a report on each paper.
\end{abstract}

\section{Creep and Plastic Flow}

From the experimental side a clear distinction was made by nearly all speakers between 'transient' and 'viscous' creep. Transient creep occurs when a metal, either a single crystal or a polycrystalline specimen, is stressed beyond the elastic limit; after an initial instantaneous extension the rate of strain $\dot{s}$ drops rapidly with increasing time $t$. Andrade ${ }^{1}$ in his pioneering work on the subject gave for polycrystalline metals the formula.

$$
\dot{s}=\beta t^{1 / 3},
$$

which ean be derived from

$$
\dot{s}=\text { const } / s^{2} \text {. }
$$

In more recent work ${ }^{2}$ on cadmium, he suggests for the flow of single crystals

$$
\dot{s}=A e^{c / s} \text {. }
$$

Orowan more recently ${ }^{3}$ has put forward the formula

$$
\dot{s}=\frac{\text { const }}{s^{2}} \exp \left(-\alpha s^{2} / k T\right)
$$

where the constants depend on the stress and temperature. It is characteristic of transient creep that : (a) it is a phenomenon taking place in single crystals or within the grains of polycrystalline materials; (b) it always takes place, even at quite low temperatures, following an instantaneous deformation of the material by any stress exceeding the elastic limit.

Viscous creep is the term given to the ultimate steady rate of creep which is obtained in certain cases. The term 'viscous' is slightly misleading, since, of course, the creep-rate is proportional to a high power of the stress. According to Andrade, in the early stages of creep there is rotation of the crystallites, accompanied by glide on crystal planes, but the final stages of true viscous creep in polycrystalline materials take place in a narrow zone surrounding the crystal boundaries, there being no deformation of the crystals as a whole at this stage

In trying to understand creep, one has first of all fairly clear evidence that slip of one crystal boundary over another can occur and is a truly viscous process, the rate being proportional to the stress. A recent paper $^{4}$ from Zener's school in Chicago has used measurements of internal damping and other anelastic effects in pure polycrystalline aluminium to obtain a figure for the rate of slip at the grain boundary in this metal. The result is

$$
\text { Rate of slip }=\text { stress } \times 5 \times 10^{8} e^{-17,250 / T} \mathrm{~cm} . / \mathrm{sec} \text {. }
$$

Of course, unless the grain boundary is flat (or of constant radius of curvature), any slip along the boundary will set up elastic strains near the surface of the grains; these strains are probably the cause of intercrystalline failure in creep tests in lead and other metals. Viscous creep possibly has its origin also in this grain boundary slip, which for large values of the applied stress will proceed until deformation takes place in the protuberances of the grain surface which lock the grains together. (This seerns to be in accord with the observations of W. A. Wood and H. J. Tapsell ${ }^{5}$, who find that a specimen of aluminium extended 0.9 per cent at $300^{\circ} \mathrm{C}$. in a creep test shows little deformation of the reflexion spots from the grains; while, after a rapid deformation followed by heat treatment at $300^{\circ} \mathrm{C}$. for an equal time, the deformation is much greater. They deduce that in the former case slip is mainly along grain boundaries; in the latter, deformation of the whole crystal occurs.) Since the stresses involved in grain-boundary slip can reach values much greater than the applied stress, one may expect deformation of the boundary region to occur until recovery or recrystallization sets in. The steady creep-rate, then, will be reached when hardening and recovery in the boundary regions just balance each other, and any quantitative theory must await a theory of these processes.

For transient creep, the elements of a theory are beginning to appear, based on the concept of dislocations. In any theory of this type, one has first to ask: Is the resistance to deformation shown by actual materials due to the difficulty of forming dislocations, or to the difficulty in moving them? The obvious fact that hardness increases with all kinds of internal strains and impurities suggests that the latter is the case. The source of dislocations remains something of a mystery; grain boundaries and mosaic boundaries (which can probably be represented as rows of dislocations) are possible sources, and, in slip bands, dislocations which have been set into rapid motion almost certainly generate others. However, for considerations of transient creep, it is probably adequate to consider that enough dislocations exist already.

One then ascribes the resistance to yield of any material to the internal stresses $\sigma_{i}$ which exist in a material due to grain boundaries, precipitates or 'stuck dislocations'. These will prevent the motion of dislocations unless the applied stress $\sigma$ exceeds $\sigma_{i}$. In this, one follows G. I. Taylor's theory of strain hardening ${ }^{6}$; but, as emphasized by the present writer, the deformation of single crystals, which harden rapidly, is theoretically a much more complicated problem than the deformation of hard materials (for example, precipitation-hardened alloys); in a hard material, investigations of creep or deformation will give information about the 
motion of dislocations under internal and external stresses which remain constant during the experiment.

According to the theory of Becker and Orowan, the energy of activation required to move a dislocation from one position of equilibrium to another, if the applied stress is $\sigma$, is

$$
V\left(\sigma-\sigma_{i}\right)^{2} / 2 G \text {. }
$$

Here $G$ is the shear modulus, and $V$ a volume of order $(200 \mathrm{~A} \text {. })^{3}$. Arguments were given by the present writer for replacing this (for precipitation-hardened materials) by

$$
U\left(\sigma_{i}\right)=0 \cdot 1 \sigma_{i} a \lambda^{2} \quad\left(1-\frac{\sigma}{\sigma_{i}}\right)^{3 / 2} .
$$

Here $a$ is the interatomic distance, and $\lambda$ the distance between centres of stress. The chance $\alpha\left(\sigma_{i}\right)$ that a dislocation moves forwards is then, per unit time,

$$
\alpha\left(\sigma_{i}\right)=v \exp \left\{-U\left(\sigma_{i}\right) / k T\right\} .
$$

As Orowan ${ }^{3}$ in particular emphasized, in transient creep, when the instantaneous extension is at an end, dislocations will be present in the material in positions for which $\sigma_{i}$ has all values from $\sigma$ upwards. Thus creep is, initially, a process of which the rate is determined by activation energies $U\left(\sigma_{i}\right)$ with all values from zero upwards. As the dislocations in particularly favourable positions, that is, those with small $U\left(\sigma_{i}\right)$, are used up, the creep-rate slows down. If $N\left(\sigma_{i}\right) d \sigma_{i}\left(\sigma_{i} \geqslant \sigma\right)$ is the number of dislocations, at the end of the instantaneous extension, in positions for which $\sigma_{i}$ lies in the range $d \sigma_{i}$, then after time $t$ the number is $N\left(\sigma_{i}\right) \exp \left\{-\alpha\left(\sigma_{i}\right) t\right\} d \sigma_{i}$. The creeprate is thus proportional to

$$
\int_{\sigma}^{\infty} N\left(\sigma_{i}\right) \alpha \exp (-\alpha t) d \sigma_{i} .
$$

On the assumption that $N\left(\sigma_{i}\right)$ is constant, this integral has been worked out by the present writer. One obtains an extension-time curve of the type

$$
S=\text { const }\{\log (v t)\}^{2 / 3},
$$

where $\nu \sim 10^{9}$ sec. $^{-1}$. Such a curve seems to represent transient creep at low temperatures (for example, for lead, Andrade, loc. cit.), but certainly does not cover the whole range of phenomena described as transient creep.

An interesting extension of the dislocation theory was given by Cottrell. Cottrell showed that impurities in solid solution would be present in abnormally low or high concentrations in the neighbourhood of a dislocation; he spoke of a "Debye-Hückel atmosphere" of dissolved atoms around a dislocation. This atmosphere, if it is given time to form, will anchor a dislocation in position, introducing a definite local value of $\sigma_{i}$ and hence accounting for the existence of a sharp yield point. The explanation was particularly apposite in the case of iron, where the yield point may be taken to depend on the segregation of the easily mobile carbon atoms around the dislocations. Snoek demonstrated to the Conference that the purest iron (less than $0 \cdot 0001$ per cent of carbon by weight) does not have a yield point. It may also be of importance in explaining the considerable observed dependence of yield point on temperature for most metals, on which Druyvesteyn reported to the Conference. It is inherent in the theory of transient creep sketched above that the activation energy $U\left(\sigma_{i}\right)$ is very large in comparison with $k T$ unless $\sigma$ is within a few per cent of $\sigma_{i}$; therefore, the dependence of yield stress on temperature cannot exceed a few per cent unless the internal stress $\sigma_{i}$ is itself temperature-dependent. Cottrell's assumption of an atmosphere of dissolved atoms in equilibrium around the dislocation will obviously lead to a decreasing resistance to yield as the temperature rises and the atmosphere is driven away. The theory also gives an elegant explanation of the phenomenon of microcreep observed by Chalmers ${ }^{7}$ in tin, where the creeprate for small stresses is proportional to stress; in this process dislocations move, carrying their atmosphere of impurity atoms with them.

\section{Internal Friction}

Snoek $^{8}$ reported on his work on the elastic aftereffect in iron; he showed that damping of vibrations in iron is partly due to the dissolved carbon atoms which set up local stresses, and which, in jumping from one equilibrium position to another, absorb energy from the vibrations. Other work on internal friction, this time on gold-copper alloys, was reported on by Guillet, who pointed out the very low damping of the alloy Cu-Au after quenching; in this quenched alloy, presumably very small tetragonal domains are found.

One can now distinguish the following mechanisms which account for internal friction: $(a)$ thermal currents between crystals of a polycrystalline material, or between strained regions of one $\operatorname{crystal}^{\circ}$; (b) slip along crystal boundarios, producing elastic strains in the adjoining material ${ }^{4}$; (c) presence of mobile dissolved atoms producing a tetragonal strain (for example, carbon in iron, as described above) ; (d) presence of mobile dislocations; internal friction of this kind is much reduced by annealing (recovery), and increased by quite small amounts of cold work ${ }^{10}$.

The cause of absorption of energy from vibrations of small amplitude is not the motion of a dislocation from one position of equilibrium to another, but its vibrations about one position of equilibrium. The amplitude of vibration (and hence the damping) should for low frequencies be proportional to $\lambda^{2}\left(\sigma / \sigma_{i}\right)^{2}$, where $\lambda$ is the distance between the centres of strain. Thus increasing hardness (increasing $\sigma_{i}$ ) gives low damping, as does also small values of $\lambda$. The small distance between the stressed tetragonal domains in the quenched $\mathrm{Cu}-\mathrm{Au}$ alloy is likely to be the cause of the abnormally low damping.

W. G. Burgersi1 discussed recovery and recrystallization, and emphasized the difference between them. Recovery seems to be connected with the motion of dislocations, which move under each other's attraction or repulsion. In recovery, individual crystals retain their identity, and lose only part of their work hardening. In recrystallization, on the other hand, new crystals, having fully the properties of the annealed material, are formed and eat up the coldworked material by a process at present little under. stood, but not so obviously connected with the motion of dislocations.

\section{Diffusion and Precipitation}

Meijering discussed the oxidation of silver containing aluminium in solid solution. Oxygen dissolves readily in silver, but the binding energy is small. The binding energy of alurninium and oxygen is large, and oxygen atoms diffusing in from the surface are trapped when they encounter aluminium atoms. A sharp boundary is formed between the interior of the specimen, which contains aluminium atoms in solid solution, and the exterior, which contains single 
molecules of aluminium oxide. These molecules are too large to diffuse through the lattice, and their binding energies are so high that they are unable to dissociate and diffuse as single atoms. Oxidation hardens the alloy, and the normal effects of overageing, leading to softening and the formation of microscopic particles of precipitate, are suppressed.

An X-ray analysis of more usual types of precipitation was reported by Guinier. His results, parts of which have already been published ${ }^{12}$, are obtained by the use of a single-crystal specimen and monochromatic radiation. In aluminium-copper alloys, where the solute atoms differ in size from those of the solute, the initial nuclei of precipitation are plates one or two atoms thick and about $25 \mathrm{~A}$. in diameter. From absolute intensity measurements he finds that about a fifth of all the copper atoms form such plates, the remainder staying in solid solution. The initial nuclei are about $90 \AA$. apart. In the aluminium-silver alloys, where all atoms are of nearly the same size, the initial nuclei are roughly spherical, and may be considered as sinusoidal fluctuations in the concentration of silver atoms with a wave-length of about $70 \mathrm{~A}$.

N. F. МотT

${ }^{1}$ Andrade, Proc. Roy. Soc., A, 84, 1 (1911); 90, 329 (1916). Andrade and Chalmers, Proc. Roy. Soc., A, 138, 348 (1932).

${ }^{2}$ Andrade and Roscoe, Proc. Phys. Soc., 49, 152 (1937).

${ }^{3}$ Orowan, $J$. West Scotland Iron and Steel Inst. (in the press).

'T'ing-Sui-Ke, Phys. Rev., 71, 533 (1947).

"Wood and Tapsell, Nature, 158, 415 (1946).

- Taylor, Proc, Roy. Soc., A, 145, 362 (1934).

'Chalmers, Proc. Roy. Soc., A, 156, 427 (1936).

Snoek, Physica, 8, 711 (1941).

'Zener, Proc. Phys. Soc., 52, 152 (1940).

${ }^{10}$ Read, Amer. Inst. Mining and Met. Eng., Tech. Pub. 1309 (1941).

11 Burgers, Proc. K. Nederl. Akad. van Wet., 50, 452 and 595 (1947).

${ }^{12}$ Guinier, J. Phys. Radium, 3, 124 (1942).

\section{THEORY AND PRACTICE OF DYEING}

$\mathrm{T}$

HE symposium held by the Society of Dyers and Colourists at Blackpool during September 25-27, to discuss recent advances in textile coloration, may prove to be of great importance, not only in the annals of the Society itself, but also in the modern history of the subject. The interests of the Society have always ranged very widely, including, at one end of the scale, the industrial production of tinctorial materials and processed fabrics, and, at the other, fundamental studies of textile fibre properties and the chemical and physical processes underlying the operations of dyeing, printing, finishing, etc. Increasingly in recent times, due principally to the growth in the physical sciences, and the narrowing specialization thereby encouraged, it has become difficult for workers situated at these antipodes to know about and to appreciate the significance of each other's work. This symposium marked a rapprochement perhaps more significant and more far-reaching than could properly be conveyed by the most careful and accurate summary of the material in the papers read and in the discussions which ensued.

The programme was almost as variegated as the interests of the members. A group of papers contributed by Dr. T. Vickerstaff and his colleagues of Imperial Chemical Industries, Ltd., by Mr. J. Crank of Courtaulds, Ltd., and by Dr. S. M. Neale and Mr. J. M. Preston of the Manchester College of Technology, attempted to define quantitatively the intimate mechanisms of dyeing processes in terms of the energy changes occurring during the processes, or by reference to the active chemical groupings and fine structure of the fibres. What may be called without disparagement the empirical technical side included an informative and well-arranged review of American and German dyeing methods by Dr. R. G. Fargher of the Shirley Institute, a discussion of automatic temperature control in dyeing machines, analytical methods for the identification and assaying of dyes, technical advances in the dyeing of acetate rayons, etc. The latter, the technical, group of papers had, of course, the wider appeal and the more general intelligibility, and they were discussed at length from all parts of the meeting. The first group, however, might fairly be said to have shown itself to be of the greater significance for future development and to have presented the most interesting, and, in the discussions that followed, the most amusing impression to the general observer.

The papers in this group were couched largely in the language and symbolism of physical chemistry, and, in spite of very ingenious interpretative efforts on the part of the protagonists, especially Dr. Neale, their direct and ultimately weighty influence on industrial procedure could not be fully appreciated by many of those whose daily concern is the bulkproduction of finished textiles for a competitive market. This situation did not, as it might have done, result in a wider cleavage, but led rather to attempts to define the function of the technologist as the agent for bringing about a liaison between the two sides, and a call for the increased intake of young trained scientific workers into the textile industries. This point of viow was put very forcefully and cogently in the discussions by Mr. R. J. Hannay, himself a chemist and calico printer of wide experience and consistently progressive attitude. What may be regarded in some degree as an admonition to both sides came from Dr. E. B. Abbot of I.C.I., who reviewed many of the recent advances in our knowledge of dyeing mechanisms, and attempted to show the extent to which this knowledge has been, or could be, made applicable in the conduct of full-scale processing operations. One most important statement is best given in Dr. Abbot's own words. "A criticism frequently levelled at research workers by technologists is that they are too far removed from realities. In the case of the direct dyes on cellulose, however, we feel that much of the research work has, on the contrary, been too closely related to narrow practical aims, and has consequently lacked that breadth of speculative perception by which primary truths are revealed. We believe the type of research carried out by Neale and his co-workers on direct dyes and cellulose to be more correctly motivated, but an extension of this work, particularly towards a wider examination of different dyes, is required." For this report to do justice, it must be said that, in the discussion, a very strong attempt was made to justify what may be called the interim type of investigation, in which technical behaviour of a range of dyes is defined quantitatively and working rules are enunciated without immediate reference to ultimate causes. In long-established industrial processes such as dyeing, where superficially simple operations exploit a very complicated interplay of variables, these interim investigations often represent the only practicable first assault on the technical problems that need to be solved. There is a danger in this type of investigation that the volume of data 